\title{
Neural Network Model for Apparent Deterministic Chaos in Spontaneously Bursting Hippocampal Slices
}

\author{
B. Biswal ${ }^{1,2}$ and C. Dasgupta ${ }^{3,4}$ \\ ${ }^{1}$ ICA1, University of Stuttgart, Pfaffenwaldring 27, D-70569 Stuttgart, Germany \\ ${ }^{2}$ Department of Physics, Sri Venkateswara College, University of Delhi, New Delhi, India \\ ${ }^{3}$ Department of Physics, Indian Institute of Science, Bangalore-560 012, India \\ ${ }^{4}$ Condensed Matter Theory Unit, JNCASR, Bangalore-560 064, India
}

\begin{abstract}
A neural network model that exhibits stochastic population bursting is studied by simulation. First return maps of interburst intervals exhibit recurrent unstable-periodic-orbit(UPO)-like trajectories similar to those found in experiments on hippocampal slices. Applications of various control methods and surrogate analysis for UPO detection also yield results similar to those of experiments. Our results question the interpretation of the experimental data as evidence for deterministic chaos and suggest caution in the use of UPO-based methods for detecting determinism in time-series data.
\end{abstract}

With recent advances in the theory of dynamical systems [1] and nonlinear time-series analysis [2], many recent studies have looked for signatures of deterministic chaos in brain dynamics [3]. This is expected to help in understanding brain functions in terms of the collective dynamics of neurons and in achieving short-term prediction and control with potential medical applications.

An experiment [4] on spontaneously bursting rat hippocampal slices bathed in a high-potassium medium has elicited much interest [5-8] in this context. In this experiment, the occurrence of recurrent trajectories resembling unstable periodic orbits (UPOs) in the first return map of interburst intervals was interpreted as evidence for chaotic dynamics. Application of chaos control [9] was found to be more effective than periodic pacing in making the bursts regular. An anticontrol method designed to make the bursts more irregular was also tested with positive results. The apparent success of these control methods was interpreted as further evidence for the occurrence of deterministic chaos. However, in subsequent work [6], similar recurrent UPO-like trajectories were also observed in simulations of a stochastic single-neuron model and comparable success in chaos control and anticontrol was reported. Recent studies $[7,8]$ in which surrogate methods are used to assess the statistical significance of UPOs claim to have reestablished the original conclusion of deterministic chaos in hippocampal burst dynamics.

We have developed and studied by simulations a simple but biologically plausible neural network model for hippocampal burst dynamics. While the variability of the interburst intervals in our model arises from stochastic transitions between two (quasi)attractors of the network dynamics, our simulations and surrogate analysis of the data yield results very similar to those reported $[4,7,8]$ for the brain slice experiment. Our work, thus, suggests that our model is appropriate for describing the experimental system and casts doubt on the interpretation of the experimental data as evidence for deterministic chaos.
The neural network model we consider is based on one developed [10] for the process of "kindling" of epilepsy. The network consists of $N$ excitatory neurons represented by the binary variables $\left\{S_{i}\right\}, i=1,2, \ldots, N$, with $S_{i}=$ 0 (1) corresponding to quiescent (firing) states. The inhibitory neurons are collectively modeled by a background inhibition assumed to be proportional to the instantaneous number of firing excitatory neurons. The "local field" $h_{i}(t)$ at the $i$ th neuron at discrete time $t$ is given by

$$
\begin{aligned}
h_{i}(t)=\sum_{j=1}^{N}[ & \left\{J_{i j} S_{j}(t)-w S_{j}(t)\right\} \\
& \left.+\lambda\left\{K_{i j} S_{j}(t-\tau)-w S_{j}(t-\tau)\right\}\right],
\end{aligned}
$$

where $w$ is the relative strength of inhibition, $\lambda$ is the relative strength of the delayed signal, and $\tau$ is the time delay associated with the delayed signal. Starting from left, the four terms in Eq. (1), respectively, represent fast recurrent excitation, fast global inhibition, slow recurrent excitation, and slow global inhibition. These four ingredients are known [11] to be essential for modeling hippocampal dynamics. Time $t$ is measured in units of "passes," each pass corresponding to random sequential updating (RSU) of $N$ neurons according to the rule: $S_{i}(t+1)=1$ if $h_{i}(t) \geq 0$ and $S_{i}(t+1)=0$ if $h_{i}(t)<0$.

Initially a fixed number $(q)$ of random low-activity patterns ("memories") $\left\{\xi_{i}^{\mu}\right\}, \quad i=1,2, \ldots, N ; \quad \mu=$ $1,2, \ldots, q$, are stored in the synaptic matrices $J_{i j}$ and $K_{i j}$ through

$$
J_{i \neq j}=\Theta\left(\sum_{\mu=1}^{q} \xi_{i}^{\mu} \xi_{j}^{\mu}\right), \quad K_{i \neq j}=\Theta\left(\sum_{\mu=1}^{q} \xi_{i}^{\mu+1} \xi_{j}^{\mu}\right),
$$

with $\xi_{i}^{q+1}=\xi_{i}^{1}, J_{i i}=K_{i i}=0$, and $\Theta(m)=1(0)$ for $m>0(m \leq 0)$. The net activity of each memory, $\sum_{i} \xi_{i}^{\mu}$, is set at a value $p \ll N$. Our simulations are carried out with the following parameter values: $N=200, q=20$, $p=10, w=0.6, \lambda=2, \tau=2$ passes. 
The fast synapses $J_{i j}$ act to stabilize the system in a memory state, the global inhibition prevents simultaneous activation of multiple memories, and the slow synapses $K_{i j}$ tend to move the network from one memory to the next one after $\tau$ passes. For $\lambda>1$, the network evolution follows a limit cycle in which all the $q$ memories are visited sequentially with the net activity $S_{\text {up }}(t)=\sum_{i=1}^{N} S_{i}(t)$ showing a low-amplitude oscillation around the average value $p$ (see Fig. 1a). To simulate the effects of the high-potassium medium, the network is "chemically kindled" through a Hebbian learning mechanism [10] switched on during 50 initial passes under reduced inhibition ( $w$ is reduced from 0.6 to 0.24 ). During this period, if $S_{i}=1$ and $S_{j}=1$ more often than 6 times over 10 consecutive passes, then the fast synaptic connection $J_{i j}$ is permanently set to 1 . Generation of these new excitatory synapses creates a new high-activity ("epileptic") attractor of the network dynamics.

The kindled network makes occasional, spontaneous, short-lived excursions to the high activity attractor (see Fig. 1b), similar to the spontaneous population bursting observed in slice experiments. The net activity is smoothened ("low-pass filtered") to $S_{s m}(t)=$ $\frac{1}{t_{s m}} \sum_{t^{\prime}=t-t_{s m}+1}^{t} S_{\text {up }}\left(t^{\prime}\right)$, with $t_{s m}=40$. The bursts appear as "spikes" in the smoothened data and the interburst intervals (IBIs) are measured from positive threshold crossings (see Fig. 1c). To prevent very rare occurrences of persistent high activity, the network is reset to a randomly chosen low-activity memory state whenever $S_{s m}(t)$ remains continuously above the threshold for more than 20 passes.

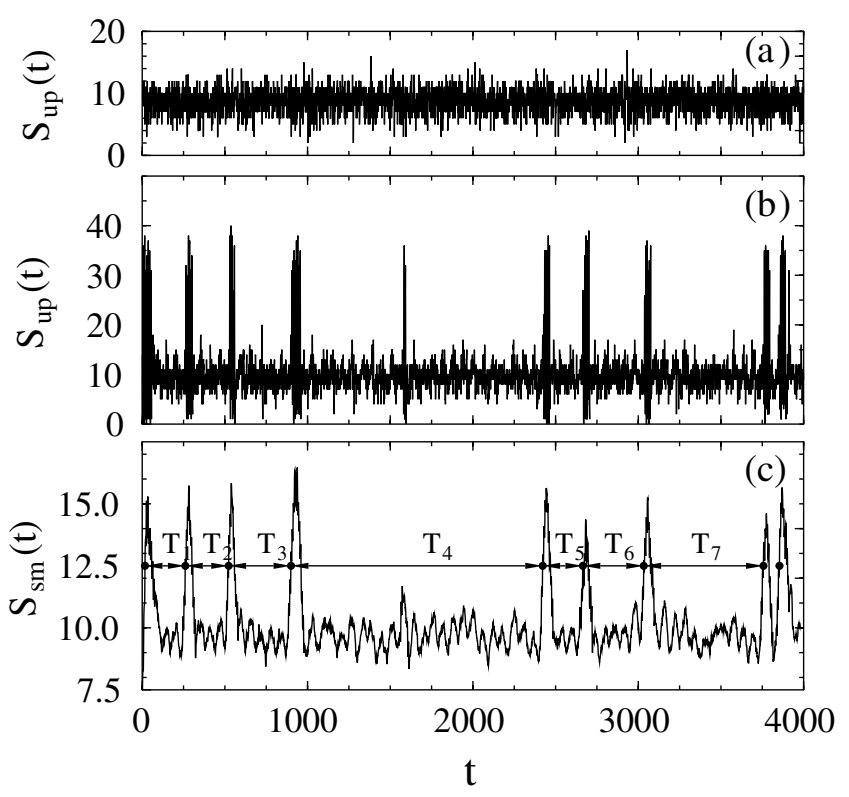

FIG. 1. (a) Network dynamics before kindling; (b) kindled network dynamics with spontaneous bursting; (c) smoothened network activity with spikes. Thresholds are denoted by dark circles and $T_{1}, T_{2}, \ldots$, are the IBIs.
The variability of the IBIs in our simulation arises entirely from the stochasticity of the sequence in which the neurons are updated. If a "deterministic" scheme in which the neurons are always updated in a (randomly chosen) fixed sequence is used, then the network remains in the low-activity state for most choices of the sequence, and exhibits periodic transitions to the high-activity attractor for certain rare sequences. Similar behavior is found for parallel updating where all the neurons are updated simultaneously. We note that the RSU scheme is the most appropriate one for modeling systems that do not have any "clocklike" mechanism.

We have studied 10 networks with different realizations of the memory states. As shown in the autocorrelation plot of Fig. 2a, the IBIs are uncorrelated in time in all the networks. Further, the probability distribution of the IBIs follows a Poisson form, $P\left(T_{n}\right) \propto \exp \left(-a T_{n}\right)$, for $T_{n}>2 t_{s m}$ (this cutoff arises from our smoothening procedure) in all the networks (see Fig. 2b). These observations strongly suggest that the spontaneous transition of the network from the low-activity state to the high-activity one is a stochastic process that has a small probability $p=1-\exp (-a)$ of occurring at each time step. As shown in Fig. 2b, the results of a Monte Carlo simulation of a Poisson process with probability $p$ of occurrence at each time step are identical to those of network simulation. Results of our studies of the divergence of two trajectories starting from neighboring points in one- and two-dimensional delay embeddings of the IBI time series are consistent with the IBIs being independent random variables.
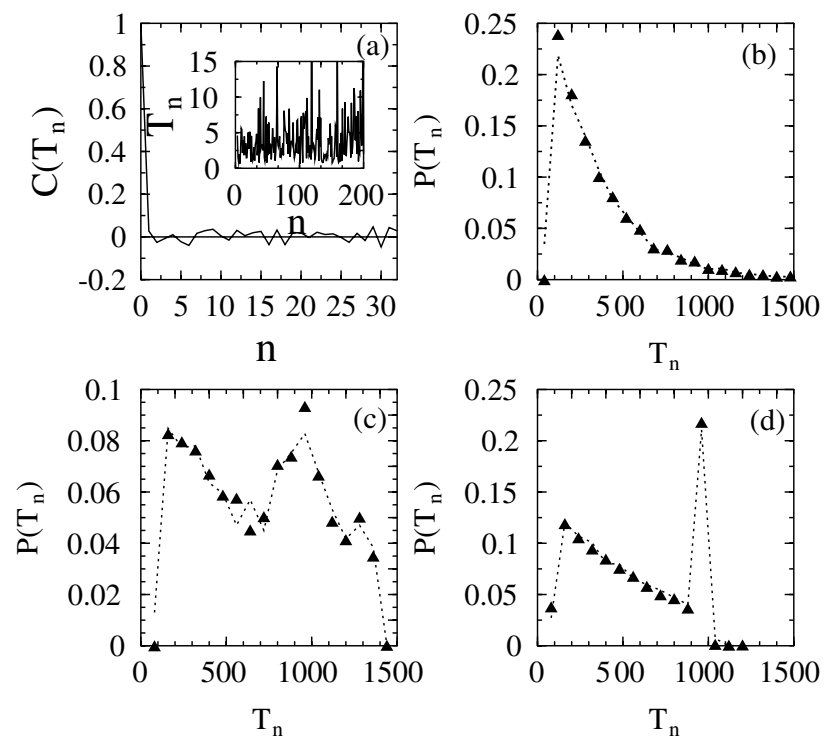

FIG. 2. (a) Autocorrelation of IBIs and sample IBI data in units of $10^{2}$ passes (inset) for network 1 in the absence of control. (b) -(d) Probability distribution of IBIs from network (dotted line) and Poisson (solid triangle) simulations for no control [network 1, panel (b)], chaos control [network 6, panel (c)], and periodic pacing [network 6, panel (d)]. 
Values of $p$ for different networks exhibit a wide variation in the range $8 \times 10^{-4}$ to $3 \times 10^{-3}$. Similar "sample-to-sample" variations and absence of correlations in the IBI time series were reported in Ref. [12] for brain slices very similar to those of Ref. [4]. The variability in the raw IBI data of Ref. [12] is qualitatively similar to that in our simulation data (see inset of Fig. 2a).

Although the bursting in our model is stochastic, first return maps of the IBIs show purely accidental occurrences [6] of UPO-like trajectories [4] that approach a fixed point $\left(T_{n}=T_{n-1}=T^{*}\right)$ along a line, $T_{n}-T^{*}=$ $s_{1}\left(T_{n-1}-T^{*}\right)$ (the stable manifold), and then diverge away along another line, $T_{n}-T^{*}=s_{2}\left(T_{n-1}-T^{*}\right)$ (the unstable manifold). In each of the 10 networks studied, many recurrent UPO-like trajectories that satisfy all the criteria adopted in [4] are found. Typical trajectories used to identify an apparent UPO and the associated manifolds are shown in Fig. 3.

Following Ref. [4], we have studied the effects of various control methods. In the proportional perturbative feedback method of chaos control (CC) [13], the system point is repeatedly placed on the stable manifold of an UPO detected during a trial period without control. This is done by generating bursts at appropriate times through external stimulation, modeled in our simulation by a reduction of the inhibition strength $w$ from 0.6 to 0.24 for five passes. Given a burst with IBI $T_{n-1}$, the external stimulation is applied $T_{n}=s_{1}\left(T_{n-1}-T^{*}\right)+T^{*}$ passes after the burst if no spontaneous burst occurs before its application. In periodic pacing (PP), the external stimulation is applied at fixed intervals of $T^{*}$ irrespective of the occurrence of spontaneous bursts, and in demand pacing (DP), the external stimulation is applied $T^{*}$ passes after a burst only if no spontaneous burst occurs prior to its application.

Typical results of the application of control, shown in Fig. 4, are qualitatively similar to those reported in

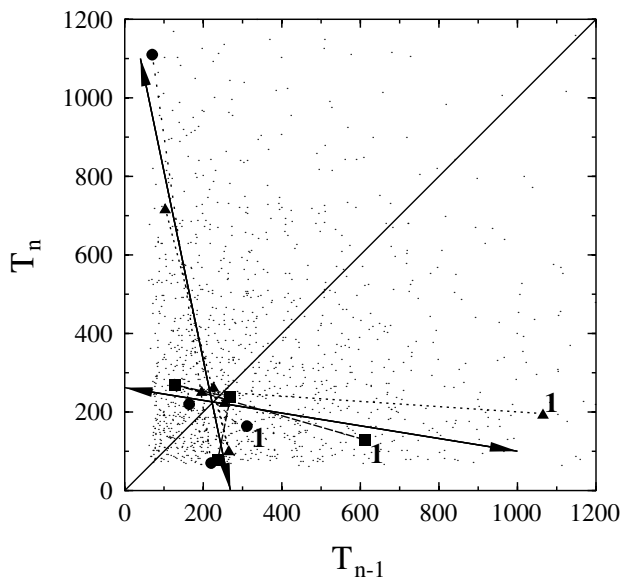

FIG. 3. Recurrent UPO-like trajectories in network 1. Three sequences (filled circles, squares, and triangles, starting points denoted by 1) starting at different times around the same stable and unstable manifolds (dark lines) are shown.
Ref. [4]. All three methods make the bursts more periodic by preventing the occurrence of large IBIs. While CC prevents the occurrence of natural IBIs above the stable manifold, PP and DP eliminate IBIs larger than $T^{*}$. This is why CC is effective when $T^{*}$ is small and the slope $s_{1}$ of the stable manifold is close to zero. In such cases, CC works better than PP because the occurrence of spontaneous bursts between two successive stimulations in PP increases the number of short IBIs. Since this does not happen in DP, this method yields the best results. For example, in network 1 for which the selected UPO, shown in Fig. 3, has $T^{*}=225, s_{1}=-0.162, s_{2}=-4.8$, the fraction of IBIs in the range $T^{*} \pm t_{s m}$, measured in 5000 IBI runs, is $0.17,0.68,0.52$, and 0.69 for no control, CC, $\mathrm{PP}$, and DP, respectively (the statistical error bars for these fractions are less than 0.01). Thus, CC and DP work better than PP (see Fig. 4) in this case. The corresponding values for network 6 , in which $T^{*}=937, s_{1}=-0.476$, $s_{2}=-1.68$, are $0.03,0.09,0.21$, and 0.4 , indicating better performance of PP and DP over CC (see Figs. 2c and $2 \mathrm{~d})$.

Even when CC works well, 70\%-90\% of the IBIs near $T^{*}$ are actually generated by the stimulation (see Fig. 4): the probability of occurrence of spontaneous IBIs near $T^{*}$ is not significantly increased during control. A similar behavior in the experiment is suggested by the experimental observation [4] that intermittent applications of the external stimulation do not produce effective control. Increasing the strength of stimulation improves control in our simulations by preventing rare failures of the stimulation to generate the required burst. This is qualitatively similar to the results of double-pulse control in the experiment. The anticontrol method proposed in Ref. [4] fails if applied over long durations ( $~ 1000$ IBIs). In applications of smaller duration ( $\sim 100$ IBIs $)$, the variability of the IBIs increases in some cases due to statistical fluctuations. In the experiment, anticontrol was applied for short durations and failed in most cases.

Our control results are similar to those reported in Ref. [6] for a stochastic single-neuron model. We have also simulated the effects of control on simple Poisson processes with $p$ determined from the network data.

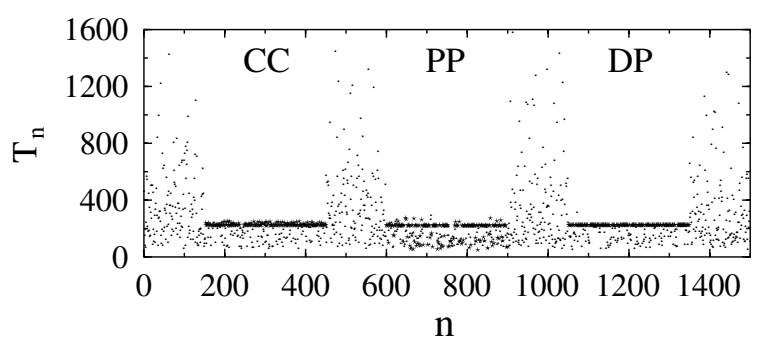

FIG. 4. Results of chaos control (CC), periodic pacing (PP), and demand pacing (DP) runs of 300 IBIs each in network 1 . No control is applied during the intermediate periods of 150 IBIs. IBIs generated by external stimulation are denoted by stars. 
The close agreement (see Figs. 2c and 2d) between the network simulation results and those of our Poisson simulations firmly establishes the stochasticity of the bursting in our model.

Recently, the statistical significance of UPOs in brain slice data has been examined $[7,8]$ using surrogate analysis of windowed IBI data sets. These studies use a dynamical transformation [7] that maps the neighborhood of an UPO in the return map to the UPO itself. First, the IBI time series $\left\{T_{n}\right\}$ is transformed by

$$
\hat{T}_{n}=\frac{T_{n+1}-s_{n}(k) T_{n}}{1-s_{n}(k)},
$$

where $\quad s_{n}(k)=\left[\left(T_{n+2}-T_{n+1}\right) /\left(T_{n+1}-T_{n}\right)\right]+$ $k\left(T_{n+1}-T_{n}\right)$. The probability distribution $\rho(\hat{T})$ of the transformed time series $\left\{\hat{T}_{n}\right\}$ is expected to show a sharp peak at an UPO, $\hat{T}=T^{*}$. Spurious peaks are eliminated [7] by averaging over many random values of $k$. Using many realizations of Gaussian-scaled phase-shuffled surrogates [14] of the original time series, the probability that the observed peaks in $\rho(\hat{T})$ could be modeled by the surrogates is estimated in the following way. From the collection of $\left\{\rho_{\text {sur }}(\hat{T})\right\}$, the ensemble average $\bar{\rho}_{\text {sur }}(\hat{T})$ is obtained. The deviation of $\rho_{\text {sur }}(\hat{T})$ for a particular surrogate from the mean $\bar{\rho}_{\text {sur }}(\hat{T})$ is measured by

$$
w(\hat{T})=\rho_{\text {sur }}(\hat{T})-\bar{\rho}_{\text {sur }}(\hat{T}) .
$$

For each of the surrogates, $W=\max (w(\hat{T}))$ is measured and the fraction $\Xi\left(W^{\prime}\right)$ of surrogates with maximum deviation $W>W^{\prime}$ is found. The statistical significance of a candidate UPO at $\hat{T}^{*}$ is estimated from the value of $\Xi(W)$ at $W=\rho\left(\hat{T}^{*}\right)-\bar{\rho}_{\text {sur }}\left(\hat{T}^{*}\right)$. In our analysis, we used 100 surrogates, $k=5 R$ where $R$ is a random number in $[1,-1]$, and 500 values of $R$. We find that this method successfully rejects the UPO-like trajectories found in the network IBIs (properly rescaled to match the analysis of Ref. [7]) only for long data sets (>1024 IBIs), but not for shorter data sets (windows). Results for a typical 128-IBI window with a "statistically significant" UPO are shown in Fig. 5. In this case, the probability of finding the peak of $\rho(\hat{T})$ (near $\hat{T}=0.5$, with $W=3.437$ ) in the surrogates is close to $4 \%$, leading to the (false) identification of a statistically significant UPO at $95 \%$ confidence level. In such analysis of many nonoverlapping windows of small width from 10 networks $(544,272$, and 136 windows of 32, 64, and 128 IBIs, respectively), statistically significant (at the 95\% confidence level) UPOs are found in $9 \%-10 \%$ of the windows. The number of networks for which such statistically significant UPOs are present in at least one of the windows is 8,10 , and 5 for window sizes 32,64 , and 128 , respectively. These numbers are very similar to those reported in Ref. [8]. Very similar results were found (e.g., 75 out of 1024 nonoverlapping 32-IBI windows showing statistically significant UPOs for network 1) in a surrogate analysis using a two-dimensional delay embedding

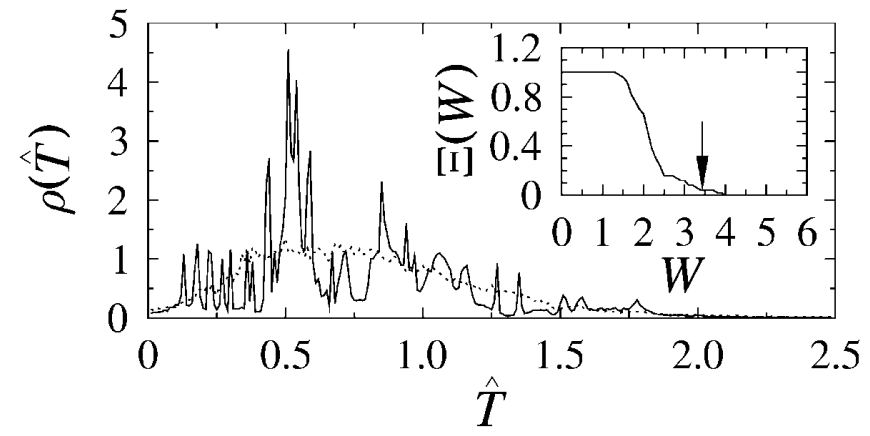

FIG. 5. The probability distributions $\rho(\hat{T})$ (solid line) and $\bar{\rho}_{\text {sur }}(\hat{T})$ (dots) for a 128 -IBI data set. Inset: The function $\Xi(W)$ used for estimating the statistical significance of UPOs. The arrow marks the maximum value of $\rho(\hat{T})-\bar{\rho}_{\text {sur }}(\hat{T})$.

[7] and both overlapping and nonoverlapping windows. Reference [8] reports the detection of statistically significant UPOs of period higher than one in intracellular data which reflect a combination of collective and single-neuron dynamics. Our network with binary neurons is not appropriate for modeling such data. Nevertheless, a similar analysis of our IBI data is planned in future work.

In conclusion, our neural network model with stochastic bursting dynamics successfully reproduces most of the findings of the brain slice experiment. This result casts doubt on the interpretation of the experimental data as evidence for deterministic chaos. Our work also shows that determinism and stochasticity need not be mutually exclusive: the evolution of our network in the low- and highactivity states is mostly deterministic, while the transitions between these two states are stochastic.

We thank Dr. G. R. Ullal for useful discussions.

[1] D. Kaplan and L. Glass, Understanding Nonlinear Dynamics (Springer, Berlin, 1998).

[2] H. Kantz and T. Schreiber, Nonlinear Time Series Analysis (Cambridge University Press, Cambridge, 1997).

[3] Nonlinear Dynamics and Brain Functions, edited by P.E. Rapp, N. Pradhan, and R. Sreenivasan (Nova Science Publishers, New York, 2000).

[4] S. J. Schiff et al., Nature (London) 370, 615 (1994).

[5] J. Glanz, Science 265, 1174 (1994); 277, 1758 (1997).

[6] D. J. Christini and J. J. Collins, Phys. Rev. Lett. 75, 2782 (1995); Chaos 7, 544 (1997).

[7] P. So et al., Phys. Rev. E 55, 5398 (1997).

[8] P. So et al., Biophys. J. 74, 2776 (1998).

[9] E. Ott, C. Grebogi, and J. A. Yorke, Phys. Rev. Lett. 64, 1196 (1990).

[10] M. R. Mehta, C. Dasgupta, and G. R. Ullal, Biol. Cybernet. 68, 335 (1993).

[11] R. D. Traub, R. Miles, and J. R. Jefferys, J. Physiology 461, 525 (1993).

[12] S. J. Schiff et al., Biophys. J. 67, 684 (1994).

[13] A. Garfinkel et al., Science 257, 1230 (1992).

[14] J. Theiler et al., Physica (Amsterdam) 58D, 77 (1992). 